OPEN ACCESS

Edited by:

Ricardo Ferraz,

University of Beira Interior, Portugal

Reviewed by:

Argyris G. Toubekis,

National and Kapodistrian University

of Athens, Greece

Cristian Alvarez,

Universidad de Los Lagos, Chile

Anthony K. May,

Deakin University, Australia

${ }^{*}$ Correspondence:

Anthony M. J. Sanchez

anthony.sanchez@univ-perp.fr

Fabio Borrani

fabio.borrani@unil.ch

tThese authors have contributed equally to this work and share senior authorship

Specialty section:

This article was submitted to

Exercise Physiology,

a section of the journal

Frontiers in Physiology

Received: 10 September 2021 Accepted: 19 November 2021

Published: 16 December 2021

Citation:

Solsona R, Berthelot H, Borrani F and Sanchez AMJ (2021) Mechanical, Cardiorespiratory, and Muscular Oxygenation Responses to Sprint Interval Exercises Under Different Hypoxic Conditions in Healthy

Moderately Trained Men.

Front. Physiol. 12:773950.

doi: 10.3389/fphys.2021.773950

\section{Mechanical, Cardiorespiratory, and Muscular Oxygenation Responses to Sprint Interval Exercises Under Different Hypoxic Conditions in Healthy Moderately Trained Men}

\author{
Robert Solsona ${ }^{1,2}$, Hugues Berthelot ${ }^{2}$, Fabio Borrani ${ }^{2 * t}$ and Anthony M. J. Sanchez ${ }^{1,2 * t}$ \\ ${ }^{1}$ University of Perpignan Via Domitia (UPVD), Faculty of Sports Sciences, Laboratoire Interdisciplinaire Performance Santé \\ Environnement de Montagne (LIPSEM), UR4640, Perpignan, France, ${ }^{2}$ Institute of Sport Sciences, University of Lausanne, \\ Lausanne, Switzerland
}

Objective: The aim of this study was to determine the effects of sprint interval exercises (SIT) conducted under different conditions (hypoxia and blood flow restriction [BFR]) on mechanical, cardiorespiratory, and muscular $\mathrm{O}_{2}$ extraction responses.

Methods: For this purpose, 13 healthy moderately trained men completed five bouts of 30 s all-out exercises interspaced by 4 min resting periods with lower limb bilateral BFR at $60 \%$ of the femoral artery occlusive pressure $\left(B R_{60}\right)$ during the first 2 min of recovery, with gravity-induced BFR (pedaling in supine position; G-BFR), in a hypoxic chamber $\left(\mathrm{FiO}_{2} \approx 13 \%\right.$; HYP) or without additional stress (NOR). Peak and average power, time to achieve peak power, rating of perceived exertion (RPE), and a fatigue index (FI) were analyzed. Gas exchanges and muscular oxygenation were measured by metabolic cart and NIRS, respectively. Heart rate $(\mathrm{HR})$ and peripheral oxygen saturation $\left(\mathrm{SpO}_{2}\right)$ were continuously recorded.

Results: Regarding mechanical responses, peak and average power decreased after each sprint $(p<0.001)$ excepting between sprints four and five. Time to reach peak power increased between the three first sprints and sprint number five $(p<0.001)$. RPE increased throughout the exercises $(p<0.001)$. Of note, peak and average power, time to achieve peak power and RPE were lower in G-BFR $(p<0.001)$. Results also showed that $\mathrm{SpO}_{2}$ decreased in the last sprints for all the conditions and was lower for HYP ( $p$ $<0.001)$. In addition, $\Delta\left[\mathrm{O}_{2} \mathrm{Hb}\right]$ increased in the last two sprints $(p<0.001)$. Concerning cardiorespiratory parameters, BFR $_{60}$ application induced a decrease in gas exchange rates, which increased after its release compared to the other conditions $(p<0.001)$. Moreover, muscle blood concentration was higher for BFR60 $(p<0.001)$. Importantly, average and peak oxygen consumption and muscular oxyhemoglobin availability during sprints decreased for HYP $(p<0.001)$. Finally, the tissue saturation index was lower in G-BFR.

Conclusions: Thus, SIT associated with G-BFR displayed lower mechanical, cardiorespiratory responses, and skeletal muscle oxygenation than the other conditions. 
Exercise with BFR60 promotes higher blood accumulation within working muscles, suggesting that BFR 60 may additionally affect cellular stress. In addition, HYP and G-BFR induced local hypoxia with higher levels for G-BFR when considering both exercise bouts and recovery periods.

Keywords: blood flow restriction (BFR), exhaustive exercise, hypoxia, oxygen extraction, skeletal muscle, supine exercise, gravity-induced blood flow restriction, vascular occlusion

\section{INTRODUCTION}

Physical exercise promotes the modulation of a large panel of cellular signaling pathways to promote metabolic and/or morphological changes that enhance performance (Bishop et al., 2019; Sanchez et al., 2019; Solsona et al., 2021). In recent years, repetition of short $(\leq 30 \mathrm{~s})$ all-out exercises were boosted in popularity because they can induce similar or higher gains of performance for a lower training volume compared to conventional endurance protocols (MacDougall et al., 1998; Barnett et al., 2004; Burgomaster et al., 2006, 2008; Gibala et al., 2006). Two distinct repeated sprint protocols are distinguished. Repeated sprint training (RST) consists of maximal sprint exercises of short duration $(\leq 10 \mathrm{~s})$ interspaced with short recovery periods (i.e., exercise to rest ratio < 1:4). On the other hand, sprint interval training (SIT) includes repeated long sprints $(\sim 30 \mathrm{~s})$ interspaced by longer rest periods ( $\sim 2 \mathrm{~min}$ ) (Buchheit and Laursen, 2013a,b; Brocherie et al., 2017). In recent years, studies have suggested that the addition of a hypoxic stimulus to chronic SIT and RST may favor several training responses (Faiss et al., 2013; Brocherie et al., 2017; Brechbuhl et al., 2020; James and Girard, 2020). For example, systemic hypoxia (HYP) may promote higher muscle perfusion and oxygenation with greater modulations of molecular adaptations (Faiss et al., 2013; Brocherie et al., 2017). These changes include increases in HIF-1 $\alpha$, myoglobin, and the expression of genes involved in mitochondrial biogenesis.

In addition to HYP, blood flow restriction (BFR) training strategies can also favor several training adaptations (Preobrazenski et al., 2021). BFR results in reduced arterial and/or venous blood flow according to the level of pressure exerted, thus generating local hypoxia and an accumulation of metabolites in the working muscles (Sugaya et al., 2011; Teixeira et al., 2018; Okita et al., 2019). BFR exercise training was found to be beneficial for enhancing adaptations such as muscle hypertrophy and aerobic capacity (Preobrazenski et al., 2021). Acute exercise with BFR may increase neuromuscular activation and type II muscle fibers recruitment (Moritani et al., 1992). In addition, BFR aerobic exercise stimulates ventilatory and cardiac response through the stimulation of group III and IV afferents (Adreani and Kaufman, 1998). These acute responses result in increased energy expenditure leading to a loss of muscle efficiency during submaximal exercise (Ozaki et al., 2010; Mendonca et al., 2014; Silva et al., 2019). However, to the best of our knowledge, BFR studies were conducted with low-intensity exercises or during RST (Willis et al., 2019), and little is known about SIT protocols using BFR.
Furthermore, different BFR models, such as cuff-, pressure chamber-, and gravity-models rise in popularity, and studies are needed to highlight their specific effects and related adaptations. Recently, a gravity-induced BFR (G-BFR) aerobic protocol has been investigated in a study conducted by Preobrazenski and colleagues (Preobrazenski et al., 2020). An ergocycle titled $45^{\circ}$ was used to generate ischemia within working muscles. In this study, muscle oxygenation was effectively found to be reduced during submaximal aerobic exercises, as previously indicated during supine exercise where faster $\mathrm{O}_{2}$ uptake kinetics was also observed (Hughson et al., 1991, 1993). Of note, Preobrazenski et al. suggested that the G-BFR model seems favorable to enhance aerobic adaptations (Preobrazenski et al., 2020). However, nothing is known about the use of such a protocol during SIT. Importantly, to our knowledge, the acute effects of HYP and BFR models on both cardiovascular and muscular responses have never been compared during SIT protocols. It appears important to understand the acute effects of these models to identify potential training adaptations for the efficient prescription of training strategies.

Thus, the goal of this study was to assess the effects of each condition on mechanical output, cardiorespiratory responses, and muscle oxygenation in healthy moderately trained men. In the present study, it has been chosen to set maximal stress for both HYP and BFR conditions that could be tolerated in this cohort in combination with the SIT protocol proposed based on preliminary work in our laboratory. Concerning the G-BFR condition, the inclined position was maintained during the entire exercise session. The hypotheses were that we would observe different responses depending on the condition with (i) a higher power output during the successive exercise bouts in NOR since the other conditions induce additional stress that may lead to a reduction of acute muscle efficiency and subsequent performance, (ii) a greater impact of HYP on the cardiorespiratory responses because HYP causes hypoxemia, and (iii) a more pronounced impact of BFR models on muscle oxygenation because they generate local hypoxia.

\section{MATERIALS AND METHODS}

\section{Subjects}

Thirteen healthy moderately trained men (mean $\pm \mathrm{SD}$, age $24 \pm 3$ years; weight $73.8 \pm 6.5 \mathrm{~kg}$; height $179 \pm 6 \mathrm{~cm}$; body fat percentage $12.5 \pm 2.1 \%$; training frequency $8 \pm 4 \mathrm{~h}$ per week) participants took part in this experiment. Prior to the first visit, the participants were informed about the experimental procedures and the possible discomforts and risks. The 
participants provided written informed consent and completed a questionnaire to exclude all potential cardiorespiratory and injury risks. The experimental protocol was approved by the local ethics committee (VD-2021-00597). All experiments were performed in accordance with the last Declaration of Helsinki.

\section{Study Design}

Participants visited the laboratory on four occasions over 4 weeks. During the 4 days of experimentation, participants performed an SIT session in different conditions, namely normal condition (NOR), with bilateral limb blood flow restriction at $60 \%$ of the total femoral artery pressure $\left(\mathrm{BFR}_{60}\right)$ during the first 2 min of recovery (Taylor et al., 2016; Mitchell et al., 2019), with G-BFR, and in a hypoxic room at $\mathrm{FiO}_{2} \approx 13 \%$ (HYP). Exercises were performed in a random order established by an independent blinded researcher. Sessions lasted between 90 and $120 \mathrm{~min}$ and were separated by at least 5 days to avoid fatigue-related interferences with the exercise sessions. Anthropometric measurements were carried out on the first visit and body fat percentage was estimated with the four skinfold thickness method (Durnin and Womersley, 1974). All tests were performed at the same time of the day to minimize the effects of circadian cycles and within similar environmental conditions. The participants were asked to maintain their dietary habits without alcohol consumption $48 \mathrm{~h}$ before each test. Athletes did not take medication or dietary supplements during the studied period. A standardized diet (55\% carbohydrate, $15 \%$ protein, and $30 \%$ fat) was proposed to the participants the day preceding each test.

\section{Determination of Femoral Artery Occlusion Pressure}

Total femoral artery occlusion pressure was measured on the day the participant followed the BFR protocol to avoid a potential effect of time and to be more accurate. The participants sat on a chair for the measurement of the total femoral artery occlusion pressure. The cuffs (SC12D, cuff size $13 \mathrm{~cm} \times 85 \mathrm{~cm}$ ) were placed around the right inferior limb proximal to the hip articulation. The occlusion pressure was progressively increased with the inflation apparatus (E20/AG101 Rapid Cuff Inflation System, D.E Hokanson Inc., Bellevue, WA, United States). The occlusion level was determined with an ultrasound linear probe (EchoWave II 3.4.4, Telemed Medical Systems, Milan, Italy) to measure blood flow. Total occlusion pressure was considered as reached when there was no detectable arterial blood flow. A total of three measurements were taken with a 1 min recovery between each evaluation. The highest-pressure value obtained was used to determine the $60 \%$ pressure applied for $\mathrm{BFR}_{60}$ during the exercise sessions. Importantly, the cuff pressure of $60 \%$ was used following preliminary work performed in the laboratory that highlighted it was the highest level that could be tolerated by the participants in combination with the present SIT protocol.

\section{Exercise Sessions}

All testing was performed in a controlled indoor environment with an ergocycle (Lode Excalibur Sport 911905, Lode B.V., Groningen, The Netherlands) programmed on constant torque mode (Wingate mode) with a torque factor of $0.8 \mathrm{Nm} \cdot \mathrm{kg}^{-1}$ The warm-up consisted of $10 \mathrm{~min}$ of cycling at $100 \mathrm{~W}(85 \mathrm{rpm})$ and two 6-s maximal sprints interspaced by a passive recovery of $54 \mathrm{~s}$. Then, after $4 \mathrm{~min}$ recovery, the participants completed five bouts of $30 \mathrm{~s}$ standing start all-out exercises interspaced by 4 min rest periods. BFR was applied with inflatable cuffs during the first 2 min of recovery after each sprint. Concerning the GBFR condition, participants maintained their inclined position during exercise bouts and recovery periods. For this condition, a structure was built to allow participants to lay horizontally on their backs as comfortably as possible. The structure also permitted handgrip to avoid body displacements during exercise. HYP was normobaric and was used during the whole session, such as the warm-up. NOR was performed below $400 \mathrm{~m}$ of altitude. The configuration (height and length) of both the saddle and handlebars was recorded to be reproduced in subsequent tests. Participants had to maintain saddle contact. They were encouraged energetically to complete every exercise maximally. Verbal indication of time was not provided to minimize pacing strategies during each sprint exercise.

Participants quoted their subjective perception of effort through the 6-20 rating perceived exertion (RPE) scale after each sprint. Individual measurements of peak $\left(P_{\text {peak }}\right)$, minimal $\left(P_{\min }\right)$, and average power, time to achieve peak power, and a fatigue index (FI) were collected. FI was calculated as follow for each sprint:

$$
F I=\frac{P_{\text {peak }}-P_{\text {min }}}{P_{\text {peak }}} \times 100
$$

During the $\mathrm{BFR}_{60}$ condition, the cuffs were placed bilaterally and proximally to the hip articulation. The cuffs were inflated during the first 2 min of recovery. The laboratory where the tests took place was below $400 \mathrm{~m}$ of altitude.

\section{Gas Exchanges and Peripheral Oxygen Saturation Measurements}

Breath-by-breath gas exchanges and peripheral oxygen saturation $\left(\mathrm{SpO}_{2}\right)$ were continuously monitored throughout the exercises and recovery periods. Oxygen consumption $\left(\dot{\mathrm{VO}}_{2}\right)$, carbon dioxide $\left(\dot{\mathrm{V} C O}_{2}\right)$ production, and minute ventilation ( $\dot{V} E)$ were measured with a gas exchange analyzer (Quark CPET, COSMED, Rome, Italy). Tidal and gas volumes $\left(\Sigma \mathrm{VT}, \Sigma \mathrm{VO}_{2}\right.$, and $\Sigma \mathrm{VCO}_{2}$ ) were cumulated for each period (exercise and recoveries). Data were treated using a second-order Butterworth filter with a cutting frequency of $0.1 \mathrm{~Hz}$. Breathing flow was measured by a bi-directional digital turbine that was calibrated using a 3-l syringe (C00600-01-11, Cosmed, Rome, Italy). A known gas mixture $\left(\mathrm{O}_{2}: 15.05 \%, \mathrm{CO}_{2}: 5.05 \%\right)$ was used to calibrate $\mathrm{O}_{2}$ and $\mathrm{CO}_{2}$ analyzers. Heart rate was collected with a Garmin monitor (HRM3-SS, Garmin, Southampton, United Kingdom). Peak and minimal values were determined for these variables during each sprint. Delta values $(\Delta)$ were calculated as the absolute difference between peak and minimal values. $\mathrm{SpO}_{2}$ was continuously recorded with a pulse oximeter (WristOx 3100, Nonin Medical Inc., Amsterdam, The 
Netherlands) and the sensor (8000Q2Sensor, Nonin Medical Inc., Amsterdam, The Netherlands) was placed at the earlobe.

\section{NIRS Measurements and Data Assessment}

Muscular $\mathrm{O}_{2}$ extraction measurements were monitored by an absolute near-infrared spectroscopy (NIRS) probe (OxiplexTS, ISS, Champagne, USA). The device was placed on the distal portion of the right vastus lateralis and was held by an elastic band wrapped around it to minimize extraneous light and movement. NIRS device includes four transmitters situated at $2.5,3.0,3.5$, and $4.0 \mathrm{~cm}$ from the receptor. The acquisition frequency was $50 \mathrm{~Hz}$ and data were averaged every $1 \mathrm{~s}$. Two different wavelength laser diodes provided the light source (682 and $834 \mathrm{~nm}$ ), and the differential pathlength factor was set to 4. Oxygen extraction was estimated by the tissue saturation index (TSI) from the NIRS measurement, which also includes total hemoglobin concentration ([tHb]), and concentrations of deoxyhemoglobin $([\mathrm{HHb}])$, and oxyhemoglobin $\left(\left[\mathrm{O}_{2} \mathrm{Hb}\right]\right)$.

\section{Statistical Analysis}

The statistical analyses were performed using Jamovi (Version 1.6.15.0). After inspecting residual plots, no obvious deviations from homoscedasticity or normality were observed. Therefore, linear mixed models were used to be more accurate with the specificity of our experimental design. Indeed, this is a longitudinal approach and linear mixed models (LMM) use mixed effect modeling to provide more precise estimates when data are hierarchized compared to repeated measures ANOVA (Gueorguieva and Krystal, 2004; Boisgontier and Cheval, 2016; Muth et al., 2016). Indeed, LMM have been developed to consider both the nested (i.e., multiple observations within a single participant in a given condition) and crossed structure (i.e., participants observed in multiple situations) of the data (Baayen et al., 2008; Boisgontier and Cheval, 2016). The flexibility of LMM makes them more appropriate for analyses of repeated measures data and when working with missing data or limited samples (Gueorguieva and Krystal, 2004; Boisgontier and Cheval, 2016; Muth et al., 2016). Conditions (i.e., NOR, BFR 60 , G-BFR, and HYP) and sprint number were the fixed effects, and the participant was set as the random effect. Post-hoc comparisons with Holm's corrections for multiple comparisons were used to adjust $p$-values. The level of significance was set at 0.05 and dispersion about the mean was expressed as SD. Effect sizes (d) (Judd et al., 2017) are provided (trivial effect $d<0.10$, small effect $0.10 \leq \mathrm{d}<0.50$, medium effect $0.50 \leq \mathrm{d}<0.80$ and large effect $\mathrm{d} \geq 0.80$ ).

\section{RESULTS}

No interaction between conditions and sprint number was found for any variable $(p>0.05)$.

\section{BFR Pressure, RPE, and $\mathrm{SpO}_{2}$}

The total occlusion pressure of the participants was $191.0 \pm 13.9$ $\mathrm{mmHg}$ and $\mathrm{BFR}_{60}$ pressure was $114.6 \pm 8.3 \mathrm{mmHg}$. A significant main effect of the condition $(p<0.01)$ and sprint number $(p$ $<0.01$ ) was found on RPE values, with higher values observed for $\mathrm{BFR}_{60}$ and HYP compared to G-BFR $(p<0.01, d=0.2$ for both). No difference was found between NOR and the other conditions for RPE. RPE increased significantly throughout the sprints $(p<0.05)$, except between sprints three and four $(p>$ $0.05)$ (Figure 1A). Concerning the average $\mathrm{SpO}_{2}$, a main effect of the condition was found $(p<0.01)$. Post-hoc analyzes revealed that $\mathrm{SpO}_{2}$ was lower for HYP compared to the other conditions $\left(p<0.01, \mathrm{~d}=-2.1,-2.5\right.$, and -1.9 for $\mathrm{BFR}_{60}, \mathrm{G}-\mathrm{BFR}$, and NOR, respectively). The values were $91 \pm 5,99 \pm 1,100 \pm 1$, and $98 \pm$ $2 \%$ for $\mathrm{HYP}, \mathrm{BFR}_{60}, \mathrm{G}-\mathrm{BFR}$, and NOR, respectively. Moreover, there was a main effect of the sprint number on $\mathrm{SpO}_{2}(p<0.01)$. $\mathrm{SpO}_{2}$ significantly decreased $(p<0.05)$ between the first and the last sprint, and between the second and the last two sprints (Figure 1B). Remarkably, $\mathrm{BFR}_{60}$ did not induce a reduction of $\mathrm{SpO}_{2}$ during its application compared to the other conditions $(p>0.05)$.

\section{Peak and Average Power Output, Time to Achieve Peak Power, and Fatigue Index}

A main effect of the condition was observed for peak power ( $p$ $<0.01)$ with a lower value found for G-BFR compared to NOR $(p<0.01, d=-1.0)$. A main effect of the sprint number was also found for peak power that decreased over time $(p<0.01)$ except between the two last sprints $(p>0.05)$. These results are presented in Figure 2A. The condition had a main effect on average power $(p<0.01)$ and post-hoc analyzes showed that values were significantly lower for G-BFR compared to the other conditions $(p<0.05, d=-0.9,-0.4$, and -0.5 for NOR, HYP, and $\mathrm{BFR}_{60}$, respectively) (Figure $2 \mathrm{~B}$ ). The sprint number also had an impact on average power as the main effect was detected $(p<0.01)$. Average power decreased over time $(p<0.01)$ except between the two last sprints $(p>0.05)$. Regarding time to achieve peak power, a main effect of the condition was observed ( $p=$ 0.02). Post-hoc analyzes revealed that the time to reach peak power was significantly greater for G-BFR compared to BFR 60 and HYP ( $p<0.05, d=1.1$, and 1.0, respectively) (Figure 2C). An effect of the sprint number was also detected for the time to achieve peak power $(p<0.01)$ that was longer in the last sprint compared to the first three sprints $(p<0.01)$. Finally, FI did not appear different depending on the condition or the sprint number $(p>0.05)$. These results are shown in Figure 2D.

\section{Gas Exchanges}

Results are presented in Table $\mathbf{1}$.

During sprints, there was a main effect of the condition on $\Sigma$ VT $(p<0.01)$. Values were lower for G-BFR compared to the other conditions $(p<0.01, d=-1.2,-1.2$, and -1.0 for NOR, HYP, and $\mathrm{BFR}_{60}$, respectively), and higher in HYP compared to $\mathrm{BFR}_{60}(p<0.05, d=0.19)$. A main effect of the condition was observed for $\dot{\mathrm{VE}}$ peak $(p<0.01)$, which was lower in G-BFR compared to the other conditions $(p<0.01, d=-1.1,-1.3$, and -0.9 for NOR, HYP, and $\mathrm{BFR}_{60}$, respectively). Values were also lower in $\mathrm{BFR}_{60}$ and NOR compared to $\operatorname{HYP}(p<0.05, d=-0.4$, and -0.1 , respectively).

Oxygen consumption showed a main effect of both conditions $(p<0.01)$ and sprint number $(p<0.01)$. Values were lower in GBFR compared to the other conditions $(p<0.01, d=-1.0,-0.7$, 


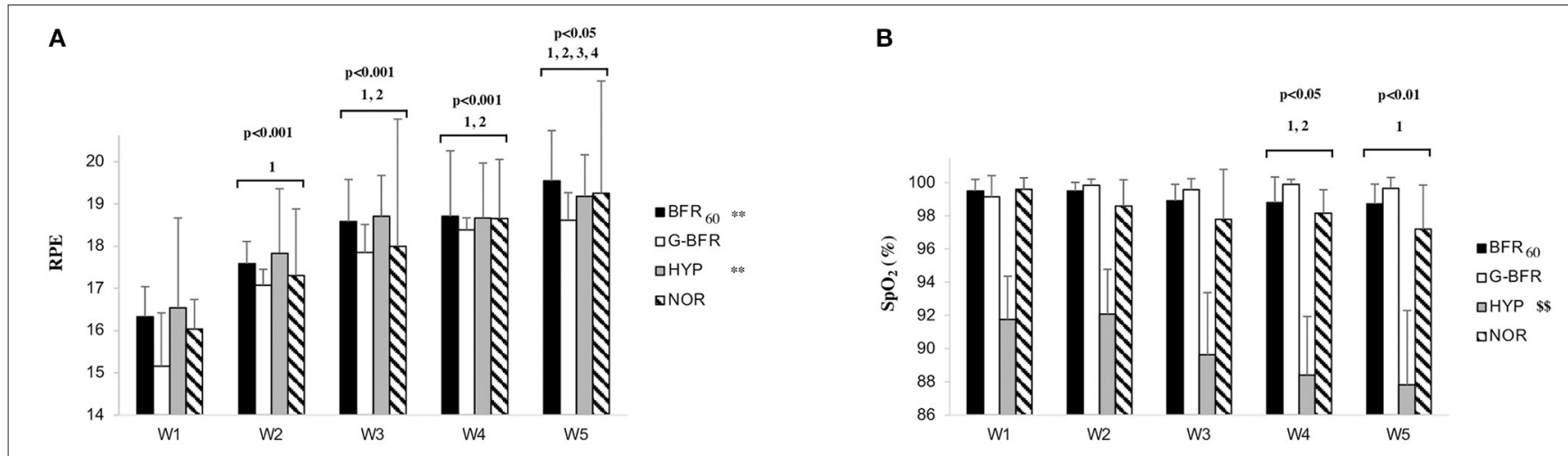

FIGURE 1 | Rating of perceived exertion (RPE) (A) and $\mathrm{SpO}_{2} \mathbf{( B )}$ values between the different conditions. BFR 60 , blood flow restriction during the two first minutes of recovery; HYP, hypoxia in a chamber at $13.0 \% \mathrm{FiO}_{2}$; G-BFR, gravity-induced BFR; NOR, control. Differences between the numbers of the sprint are highlighted by numbers and symbols are used for differences between conditions. ${ }^{1}$ Different from sprint number one; ${ }^{2}$ different from the second sprint; ${ }^{3}$ different from the third sprint; ${ }^{4}$ different from the fourth sprint, these differences concern the effect of sprint number without distinguishing the exercise modality (n.b., no statistical interaction was found); ${ }^{* \star} p<0.01$ different from G-BFR; ${ }^{\$} \$ p<0.01$ different from the other conditions.

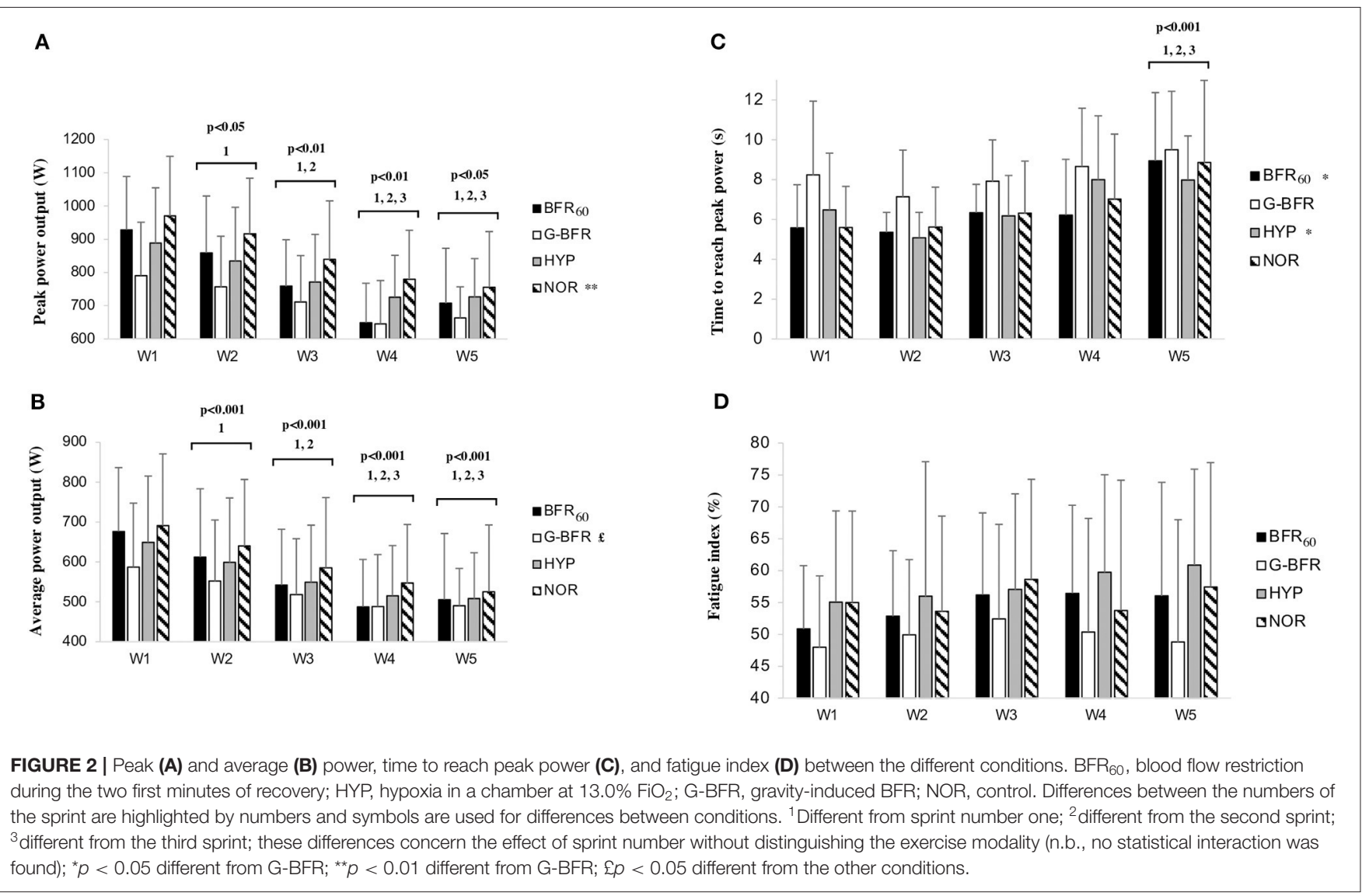

and -0.8 for NOR, HYP, and $\mathrm{BFR}_{60}$, respectively) and in HYP compared to NOR $(p<0.01, d=-0.5)$. We found main effects reflecting a difference on $\dot{\mathrm{V}}_{2 \text { peak }}$ depending on the condition ( $p$ $<0.01)$, and the sprint number $(p<0.01)$. Values were lower in HYP compared to all conditions ( $p<0.05, d=-1.2,-0.8$, and -0.9 for HYP, $\mathrm{BFR}_{60}$, and G-BFR, respectively), and higher in NOR compared to $\mathrm{BFR}_{60}$ and G-BFR $(p<0.05, d=0.3$, and 0.2 , respectively). Regarding the sprint number, it was higher in the second sprint compared to the fourth and fifth $(p<0.01)$ : 3932 $\pm 248,3740 \pm 316$, and $3656 \pm 298 \mathrm{ml} \cdot \mathrm{min}^{-1}$, respectively.

Finally, mean sprint heart rate (HR) presented a condition main effect $(p<0.01)$ and a sprint number main effect $(p<$ $0.01)$. HR was lower in G-BFR $(p<0.01, d=-0.8)$ and in $\mathrm{BFR}_{60}(p<0.05, d=-0.3)$ compared to NOR. HR was also 
TABLE 1 | Cardio-respiratory responses during the interventions.

\begin{tabular}{|c|c|c|c|c|}
\hline & $\mathrm{BFR}_{60}$ & G-BFR & HYP & NOR \\
\hline \multicolumn{5}{|l|}{ Exercise } \\
\hline$\Sigma \mathrm{VT}(\mathrm{L})$ & $58.2 \pm 15.2$ & $43.7 \pm 13.5^{\ddagger \ddagger}$ & $61.1 \pm 15.1^{\ddagger \star \star}$ & $61.0 \pm 15.9^{\star \star}$ \\
\hline$\dot{\mathrm{V}} \mathrm{E}_{\min }\left(\mathrm{L} \cdot \mathrm{min}^{-1}\right)$ & $39.3 \pm 11.9$ & $29.3 \pm 11.2^{\ddagger \ddagger}$ & $36.0 \pm 14.3^{\star \star}$ & $38.5 \pm 12.4^{\star \star}$ \\
\hline$\Delta \dot{\mathrm{V}} \mathrm{E}\left(\mathrm{L} \cdot \mathrm{min}^{-1}\right)$ & $111.2 \pm 24.3$ & $95.5 \pm 20.4^{\ddagger \ddagger}$ & $125.9 \pm 24.7^{\ddagger \star \star}$ & $119.2 \pm 24.2^{\star \star \$}$ \\
\hline$\dot{\mathrm{V}} \mathrm{O}_{2 \min }\left(\mathrm{mL} \cdot \mathrm{min}^{-1}\right)$ & $663.9 \pm 171.4$ & $562.9 \pm 144.3^{\ddagger \ddagger}$ & $609.1 \pm 199.8$ & $665.5 \pm 179.5^{\star \star}$ \\
\hline$\Delta \dot{\mathrm{V}} \mathrm{O}_{2}\left(\mathrm{~mL} \cdot \mathrm{min}^{-1}\right)$ & $3202.0 \pm 637.8$ & $3332.5 \pm 589.5$ & $2812.9 \pm 388.8^{\ddagger \ddagger \star \star}$ & $3368.2 \pm 483.0^{\neq \$ \$}$ \\
\hline$\Sigma \mathrm{VCO}_{2}(\mathrm{~L})$ & $1.106 \pm 0.230$ & $0.874 \pm 0.193^{\ddagger \ddagger}$ & $1.015 \pm 0.198^{\ddagger \ddagger \star \star}$ & $1.110 \pm 0.228^{\star \star \$ \$}$ \\
\hline$\dot{\mathrm{V}} \mathrm{CO}_{2 \text { peak }}\left(\mathrm{mL} \cdot \mathrm{min}^{-1}\right)$ & $3105.1 \pm 828.8$ & $3109.7 \pm 713.5$ & $3046.8 \pm 734.8$ & $3241.1 \pm 830.0^{\$}$ \\
\hline$\dot{\mathrm{V}} \mathrm{CO}_{2 \min }\left(\mathrm{mL} \cdot \mathrm{min}^{-1}\right)$ & $835.3 \pm 268.6$ & $675.8 \pm 215.7^{\ddagger \ddagger}$ & $659.9 \pm 229.4^{\ddagger \ddagger}$ & $803.3 \pm 244.2^{\star \star \$ \$}$ \\
\hline $\mathrm{HR}_{\min }(\mathrm{bpm})$ & $113.3 \pm 21.2$ & $101.8 \pm 20.7^{\ddagger \ddagger}$ & $115.8 \pm 21.3^{\star *}$ & $117.7 \pm 21.2^{\star \star}$ \\
\hline$\Delta \mathrm{HR}(\mathrm{bpm})$ & $48.8 \pm 20.1$ & $54.6 \pm 20.5^{\ddagger \ddagger}$ & $47.3 \pm 18.3^{\star \star}$ & $48.0 \pm 18.7^{\star \star}$ \\
\hline \multicolumn{5}{|c|}{ Recovery periods (mean values) } \\
\hline$\Sigma \mathrm{VT}(\mathrm{L})$ & $281.1 \pm 70.8$ & $239.0 \pm 67.0^{\ddagger \ddagger}$ & $308.4 \pm 71.3^{\ddagger \neq \star \star}$ & $291.9 \pm 61.9^{\star \star \$ \$}$ \\
\hline$\Sigma \bigvee_{2}(\mathrm{~L})$ & $5.6 \pm 0.9$ & $5.5 \pm 0.8$ & $5.9 \pm 0.8^{\ddagger \ddagger \star \star}$ & $5.9 \pm 0.8^{\ddagger \ddagger \star \star}$ \\
\hline$\Sigma \mathrm{VCO}_{2}(\mathrm{~L})$ & $6.2 \pm 1.5$ & $6.3 \pm 1.3$ & $6.3 \pm 1.3$ & $6.6 \pm 1.3$ \\
\hline $\mathrm{HR}(\mathrm{bpm})$ & $137.1 \pm 16.1$ & $123.6 \pm 18.6^{\ddagger}$ & $139.5 \pm 18.1^{\star \star}$ & $139.2 \pm 16.4^{\star \star}$ \\
\hline $\mathrm{HR}_{\text {peak }}(\mathrm{bpm})$ & $167 \pm 12$ & $164 \pm 12$ & $169 \pm 12^{\ddagger \star \star}$ & $171 \pm 10^{\ddagger \ddagger \star \star}$ \\
\hline $\mathrm{HR}_{\min }(\mathrm{bpm})$ & $116 \pm 20$ & $104 \pm 20^{\ddagger}$ & $118 \pm 22^{\star \star}$ & $119 \pm 19^{\star \star}$ \\
\hline$\Delta \mathrm{HR}(\mathrm{bpm})$ & $51 \pm 19$ & $59 \pm 16^{\ddagger}$ & $50 \pm 16^{\star *}$ & $52 \pm 15^{\star \star}$ \\
\hline \multicolumn{5}{|l|}{ Recovery periods } \\
\hline$\Sigma \mathrm{VCO}_{2}(\mathrm{~L})$ & $2.537 \pm 0.528$ & $2.065 \pm 0.446^{\ddagger \ddagger}$ & $2.086 \pm 0.434^{\ddagger \ddagger}$ & $2.263 \pm 0.464^{\ddagger \neq * \$}$ \\
\hline
\end{tabular}

$B F R_{60}$, blood flow restriction during the two first minutes of recovery; HYP, a hypoxic condition in a chamber $\left(F_{i O}{ }_{2} 13.0 \%\right) ;$ G-BFR, gravity-induced BFR; NOR, control; $\Sigma V T$, cumulated ventilation; $\dot{V} E_{\text {peak, }}$, peak minute ventilation; $\dot{V} E_{\text {min }}$, minimum minute ventilation; $\triangle \dot{V} E$, $\left|\dot{V} E_{\text {peak }}-\dot{V} E_{\text {min }}\right| ; \Sigma V O_{2}$, cumulated oxygen consumption; $\dot{V} O_{2 p e a k}$, peak oxygen consumption rate; $\dot{V} \mathrm{O}_{2 m i n}$, mean oxygen consumption rate; $\Delta \dot{V} \mathrm{O}_{2},\left|\dot{V} \mathrm{~V}_{2 \text { peak }}-\dot{\mathrm{V}} \mathrm{O}_{2 \text { min }}\right| ; \Sigma V \mathrm{VO}_{2}$, cumulated carbon dioxyde release; $\dot{V} \mathrm{CO}_{2}$ peak, peak carbon dioxyde release rate; $\dot{V} \mathrm{CO}_{2}$ min, minimum carbon dioxyde release rate; $\Delta \dot{V} \mathrm{CO}_{2},\left|\dot{V} \mathrm{CO}_{2 \text { peak }}-\dot{V} \mathrm{CO}_{2 \text { min }}\right| ; H R_{\text {mean }}$, mean heart rate; $H R_{\text {peak, }}$, peak heart rate; $H R_{\text {min }}$, minimum heart rate; $\Delta H R,\left|H R_{\text {peak }}-H R_{\text {min }}\right|$. Data are expressed as mean $\pm S D$. "Significantly different from G-BFR; ${ }^{*}$ significantly different from BFR ${ }_{60} ;{ }^{\$}$ significantly different from HYP. The statistical significance threshold is set at $p<0.05$ (one symbol) and $p<0.01$ (two symbols).

lower in the first sprint compared to the other sprints, and in the second sprint compared to the third and the fifth ones $(p<$ 0.05): $128.4 \pm 5.8,136.8 \pm 5.2,141.3 \pm 5.5$, and $143.1 \pm 4.4 \mathrm{bpm}$ respectively. Only a condition main effect was found for $\mathrm{HR}_{\text {peak }}$ $(p<0.01)$. Values were lower in G-BFR compared to the other conditions $(p<0.05, d=-0.8,-0.5$, and -0.4 for NOR, HYP, and $\mathrm{BFR}_{60}$, respectively) and in $\mathrm{BFR}_{60}$ compared to $\mathrm{NOR}(p<$ $0.05, d=-0.4)$.

\section{Muscular $\mathrm{O}_{2}$ Extraction}

Results are presented in Table 2, Figure 3.

We found a significant condition main effect on TSI $(p<$ 0.01). TSI was higher in $\mathrm{BFR}_{60}$ compared to HYP and G-BFR
( $p<0.01, d=0.8$, and 1.3, respectively), and lower in G-BFR compared to the other conditions $(p<0.01, d=-0.9,-0.5$, and -1.3 for NOR, HYP, and $\mathrm{BFR}_{60}$, respectively). Mean session (both sprint and recovery periods included) TSI was lower in GBFR compared to the other conditions $(p<0.05)$. A main effect of condition was detected for $[\mathrm{tHb}],[\mathrm{tHb}]_{\max }$, and $[\mathrm{tHb}]_{\min }(p$ $<0.01)$. Values were higher in $\mathrm{BFR}_{60}$ compared to the other conditions $\left(p<0.01,[\mathrm{tHb}]: d=0.4,0.7\right.$, and 0.7 ; $[\mathrm{tHb}]_{\max }: d$ $=0.5,0.6$, and $0.7 ;[\mathrm{tHb}]_{\min }: d=0.4,0.6$, and 0.7 for NOR, HYP, and G-BFR, respectively).

$\left[\mathrm{O}_{2} \mathrm{Hb}\right]_{\max },\left[\mathrm{O}_{2} \mathrm{Hb}\right]_{\min }$, and $\left[\mathrm{O}_{2} \mathrm{Hb}\right]$ also presented a main effect of condition $(p<0.01)$. Values were higher in $\mathrm{BFR}_{60}$ compared to the other conditions $\left(p<0.05,\left[\mathrm{O}_{2} \mathrm{Hb}\right]_{\max }: d=\right.$ 
TABLE 2 | Near-infrared spectroscopy (NIRS) parameters during the interventions.

\begin{tabular}{|c|c|c|c|c|}
\hline & $\mathrm{BFR}_{60}$ & G-BFR & HYP & NOR \\
\hline \multicolumn{5}{|l|}{ Exercise } \\
\hline TSI (\%) & $63.7 \pm 6.3$ & $54.0 \pm 8.2^{\ddagger \ddagger}$ & $58.2 \pm 8.0^{\ddagger \neq \star \star}$ & $61.0 \pm 6.9^{* *}$ \\
\hline $\mathrm{TSI}_{\max }(\%)$ & $80.7 \pm 6.4$ & $72.3 \pm 10.9$ & $78.8 \pm 3.8^{\star \star}$ & $80.7 \pm 4.6^{\star \star}$ \\
\hline TSI $I_{\min }(\%)$ & $56.0 \pm 8.1$ & $46.4 \pm 10.0^{\ddagger}$ & $49.8 \pm 11.1^{\ddagger \star}$ & $52.0 \pm 11.2^{\ddagger \star}$ \\
\hline$\Delta \mathrm{TSI}(\%)$ & $24.7 \pm 9.4$ & $25.9 \pm 10.5$ & $29.0 \pm 11.5$ & 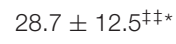 \\
\hline$[\mathrm{tHb}](\mu \mathrm{M})$ & $126.6 \pm 35.1$ & $105.7 \pm 18.4^{\ddagger \ddagger}$ & $107.1 \pm 22.3^{\ddagger \ddagger}$ & $113.2 \pm 25.6^{\ddagger \ddagger}$ \\
\hline$[\mathrm{tH} \mathrm{Hb}]_{\max }(\mu \mathrm{M})$ & $135.9 \pm 38.1$ & $113.5 \pm 19.9^{\ddagger \ddagger}$ & $115.6 \pm 25.8^{\ddagger \ddagger}$ & $120.0 \pm 27.3^{\ddagger \ddagger}$ \\
\hline$[\mathrm{tH} b]_{\min }(\mu \mathrm{M})$ & $120.1 \pm 33.8$ & $101.0 \pm 17.1^{\ddagger \ddagger}$ & $102.3 \pm 20.4^{\ddagger \ddagger}$ & $107.9 \pm 23.8^{\ddagger \ddagger}$ \\
\hline$\Delta[\mathrm{tHb}](\mu \mathrm{M})$ & $15.7 \pm 11.5$ & $12.5 \pm 4.6$ & $13.2 \pm 7.4$ & $12.1 \pm 7.2$ \\
\hline$\left[\mathrm{O}_{2} \mathrm{Hb}\right](\mu \mathrm{M})$ & $81.0 \pm 25.7$ & $56.7 \pm 11.3^{\ddagger}$ & $61.7 \pm 11.7^{\ddagger}$ & $68.1 \pm 11.7^{\ddagger \star s}$ \\
\hline$\left[\mathrm{O}_{2} \mathrm{Hb}\right]_{\max }(\mu \mathrm{M})$ & $108.9 \pm 36.5$ & $89.7 \pm 21.3^{\ddagger}$ & $81.0 \pm 19.8^{\ddagger}$ & $94.8 \pm 21.3^{\ddagger \star}$ \\
\hline$\left[\mathrm{O}_{2} \mathrm{Hb}\right]_{\min }(\mu \mathrm{M})$ & $68.9 \pm 23.3$ & $47.0 \pm 10.5^{\ddagger}$ & $50.9 \pm 11.8^{\ddagger}$ & $56.2 \pm 11.5^{\ddagger \star}$ \\
\hline$\Delta\left[\mathrm{O}_{2} \mathrm{Hb}\right](\mu \mathrm{M})$ & $40.0 \pm 20.6$ & $33.9 \pm 13.7$ & $38.8 \pm 21.4$ & $38.6 \pm 22.5$ \\
\hline$[\mathrm{HHb}](\mu \mathrm{M})$ & $45.6 \pm 14.4$ & $49.0 \pm 14.5$ & $45.4 \pm 16.2$ & $5.4 \pm 16.2$ \\
\hline$[\mathrm{HHb}]_{\max }(\mu \mathrm{M})$ & $55.6 \pm 18.9$ & $57.6 \pm 18.9$ & $54.8 \pm 20.9$ & $56.5 \pm 24.7$ \\
\hline$[\mathrm{HHb}]_{\min }(\mu \mathrm{M})$ & $24.4 \pm 8.4$ & $30.2 \pm 12.5$ & $23.7 \pm 6.6^{\star \star}$ & $22.6 \pm 8.3^{\star \star}$ \\
\hline$\Delta[\mathrm{HHb}](\mu \mathrm{M})$ & $31.2 \pm 18.0$ & $27.4 \pm 15.3$ & $31.1 \pm 17.0$ & $34.0 \pm 21.8^{\star \star}$ \\
\hline \multicolumn{5}{|c|}{ Recovery periods } \\
\hline \multicolumn{5}{|l|}{ First 2 min } \\
\hline TSI (\%) & $72.5 \pm 6.6$ & $65.9 \pm 10.9$ & $73.7 \pm 4.5$ & $77.3 \pm 3.0^{\star \star}$ \\
\hline $\mathrm{TSI}_{\max }(\%)$ & $83.4 \pm 5.6$ & $8.7 \pm 9.2$ & $82.3 \pm 3.2$ & $84.4 \pm 3.2$ \\
\hline $\mathrm{TSI}_{\min }(\%)$ & $54.6 \pm 8.0$ & $45.7 \pm 14.4$ & $50.8 \pm 9.8$ & $53.0 \pm 7.9$ \\
\hline$\Delta \mathrm{TSI}(\%)$ & $28.8 \pm 9.1$ & $33.0 \pm 13.5$ & $31.5 \pm 10.3$ & $31.5 \pm 8.5$ \\
\hline$[\mathrm{tHb}](\mu \mathrm{M})$ & $146.6 \pm 52.6$ & $113.2 \pm 20.8$ & $113.8 \pm 2$ & $123.5 \pm 30.2$ \\
\hline$[\mathrm{tHb}]_{\max }(\mu \mathrm{M})$ & $178.5 \pm 72.7$ & $121.7 \pm 24.2^{\ddagger}$ & $123.2 \pm 29.0^{\ddagger}$ & $140.4 \pm 46.8$ \\
\hline$[\mathrm{tH}-\mathrm{b}]_{\min }(\mu \mathrm{M})$ & $111.0 \pm 41.0$ & $99.8 \pm 20.5$ & $100.7 \pm 21.8$ & $105.8 \pm 23.0$ \\
\hline$\Delta[\mathrm{tH} b](\mu \mathrm{M})$ & $67.5 \pm 39.6$ & $21.9 \pm 11.8^{\ddagger}$ & $22.5 \pm 9.7^{\ddagger}$ & $34.6 \pm 30.0^{\ddagger}$ \\
\hline$\left[\mathrm{O}_{2} \mathrm{Hb}\right](\mu \mathrm{M})$ & $108.5 \pm 47.6$ & $74.7 \pm 18.2^{\ddagger}$ & $84.3 \pm 19.7$ & $5.5 \pm 23.3$ \\
\hline$\left[\mathrm{O}_{2} \mathrm{Hb}\right]_{\max }(\mu \mathrm{M})$ & & & & 39.5 \\
\hline$\left[\mathrm{O}_{2} \mathrm{Hb}\right]_{\min }(\mu \mathrm{M})$ & $67.1 \pm 27.1$ & $47.9 \pm 14.8$ & $52.4 \pm 11.2$ & $58.7 \pm 9.3$ \\
\hline$\Delta\left[\mathrm{O}_{2} \mathrm{Hb}\right](\mu \mathrm{M})$ & $73.9 \pm 49.5$ & $44.7 \pm 23.7$ & $47.2 \pm 2$ & $57.7 \pm 33.4$ \\
\hline$[\mathrm{HHb}](\mu \mathrm{M})$ & $39.1 \pm 12.7$ & $39.3 \pm 14.2$ & $29.6 \pm 7.6$ & $28.0 \pm 8.4^{\ddagger}$ \\
\hline$[\mathrm{HH} \mathrm{Hb}]_{\max }(\mu \mathrm{M})$ & $61.3 \pm 22.1$ & $61.5 \pm 24.9$ & $54.9 \pm 20.3$ & $56.5 \pm 24.7$ \\
\hline$[\mathrm{HHb}]_{\min }(\mu \mathrm{M})$ & $21.8 \pm 7.3$ & $9 \pm 9.2$ & $0.4 \pm 4.5$ & $9.8 \pm 6.9$ \\
\hline$\Delta[\mathrm{HHb}](\mu \mathrm{M})$ & $39.5 \pm 18.8$ & & $34.5 \pm 17.7$ & $36.6 \pm 21.6$ \\
\hline \multicolumn{5}{|l|}{ Last 2 min } \\
\hline TSI (\%) & $77.7 \pm 6.7$ & $73.0 \pm 10.6$ & $79.5 \pm 4.1$ & $81.6 \pm 3.6^{*}$ \\
\hline $\mathrm{TSI}_{\max }(\%)$ & $84.5 \pm 5.1$ & $9.1 \pm 8.8$ & $2.3 \pm 3.2$ & $84.3 \pm 3.5$ \\
\hline $\mathrm{TSI}_{\min }(\%)$ & $62.7 \pm 13.5$ & $63.0 \pm 14.5$ & $75.0 \pm 6.0^{\ddagger \star}$ & $77.7 \pm 6.1^{\ddagger *}$ \\
\hline$\Delta \mathrm{TSI}(\%)$ & $21.8 \pm 12.0$ & $6.1 \pm 10.2$ & 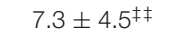 & $6.6 \pm 3.9^{\ddagger \ddagger *}$ \\
\hline$[\mathrm{tH} b](\mu \mathrm{M})$ & $136.7 \pm 49.7$ & $113.7 \pm 20.3$ & $114.5 \pm 24.9$ & $123.9 \pm 28.0$ \\
\hline$[\mathrm{tHb}]_{\max }(\mu \mathrm{M})$ & $167.2 \pm 65.4$ & $120.6 \pm 23.6^{\ddagger}$ & $121.1 \pm 27.4$ & $136.6 \pm 41.0$ \\
\hline$[\mathrm{tH}-\mathrm{b}]_{\min }(\mu \mathrm{M})$ & $112.7 \pm 43.9$ & $104.6 \pm 21.8$ & $105.8 \pm 21.9$ & $111.4 \pm 23.2$ \\
\hline$\Delta[\mathrm{tHb}](\mu \mathrm{M})$ & $54.5 \pm 33.9$ & $16.0 \pm 12.8^{\ddagger \ddagger}$ & $15.4 \pm 7.3^{\ddagger \ddagger}$ & $25.2 \pm 23.9^{\ddagger \ddagger}$ \\
\hline$\left[\mathrm{O}_{2} \mathrm{Hb}\right](\mu \mathrm{M})$ & $108.4 \pm 45.8$ & $83.3 \pm 20.2$ & $91.5 \pm 22.6$ & $100.8 \pm 21.3$ \\
\hline$\left[\mathrm{O}_{2} \mathrm{Hb}\right]_{\max }(\mu \mathrm{M})$ & $136.4 \pm 62.3$ & $93.1 \pm 23.6$ & $99.0 \pm 25.4$ & $113.7 \pm 34.8$ \\
\hline$\left[\mathrm{O}_{2} \mathrm{Hb}\right]_{\min }(\mu \mathrm{M})$ & $79.6 \pm 42.4$ & $70.9 \pm 22.1$ & $82.3 \pm 19.2$ & $88.9 \pm 16.0$ \\
\hline$\Delta\left[\mathrm{O}_{2} \mathrm{Hb}\right](\mu \mathrm{M})$ & $56.9 \pm 41.1$ & $22.2 \pm 16.9^{\ddagger}$ & $16.7 \pm 9.1^{\ddagger \ddagger}$ & $24.8 \pm 22.9^{\ddagger}$ \\
\hline$[\mathrm{HHb}](\mu \mathrm{M})$ & $28.3 \pm 11.6$ & $30.4 \pm 12.9$ & $23.0 \pm 5.7$ & $23.0 \pm 8.8$ \\
\hline
\end{tabular}

(Continued)
TABLE 2 | Continued

\begin{tabular}{lcccc}
\hline & BFR $_{60}$ & G-BFR & HYP & NOR \\
\hline$[\mathrm{HHb}]_{\max }(\mu \mathrm{M})$ & $45.8 \pm 21.6$ & $38.9 \pm 19.6$ & $26.5 \pm 7.7^{\ddagger}$ & $26.3 \pm 9.5^{\ddagger}$ \\
{$[\mathrm{HHb}]_{\min }(\mu \mathrm{M})$} & $19.2 \pm 6.6$ & $23.8 \pm 9.7$ & $20.2 \pm 4.6$ & $20.1 \pm 7.8$ \\
$\Delta[\mathrm{HHb}](\mu \mathrm{M})$ & $26.6 \pm 16.5$ & $15.1 \pm 12.3^{\ddagger}$ & $6.2 \pm 4.3^{\ddagger \ddagger}$ & $6.2 \pm 3.0^{\ddagger \ddagger}$ \\
\hline
\end{tabular}

$B F R_{60}$, blood flow restriction during the two first minutes of recovery; HYP, hypoxic condition in a chamber $\left(\mathrm{FiO}_{2}\right.$ 13.0\%); G-BFR, gravity-induced BFR; NOR, control; TSI, tissue saturation index; TSImax, maximum TSI; TSImin, minimum TSI; $\triangle T S I$, $\left|T S I_{\max }-T S I_{\min }\right| ;[t H b]$, total hemoglobin content; [HHb] $]_{\max }$, maximum [tHb]; $[t H b]_{\min }$, minimum $[t H b] ; \Delta[t H b],\left|[t H b]_{\max }-[t H b]_{\min }\right| ;\left[\mathrm{O}_{2} \mathrm{Hb}\right]$, oxyhaemoglobin content; $\left[\mathrm{O}_{2} \mathrm{Hb}\right]_{\max }$, maximum $\left[\mathrm{O}_{2} \mathrm{Hb}\right] ;\left[\mathrm{O}_{2} \mathrm{Hb}\right]_{\min }$, minimum $\left.\left[\mathrm{O}_{2} \mathrm{Hb}\right] ; \Delta_{2} \mathrm{O}_{2} \mathrm{Hb}\right],\left[\mathrm{O}_{2} \mathrm{Hb}\right]_{\max }-\left[\mathrm{O}_{2} \mathrm{Hb}\right]_{\min } \mid ;[\mathrm{HHb}]$, deoxyhaemoglobin content; $[\mathrm{HH} b]_{\max }$, maximum $[\mathrm{HHb}] ;[\mathrm{HHb}]_{\min }$, minimum $[\mathrm{HHb}] ;$ $\Delta[H H b],\left|[H H b]_{\max }-[H H b]_{\min }\right|$. Data are expressed as mean $\pm S D$. " Significantly different from G-BFR; " ${ }^{*}$ significantly different from BFR60; ${ }^{\$}$ significantly different from HYP. Statistical significance threshold is set at $p<0.05$ (one symbol) and $p<0.01$ (two symbols).

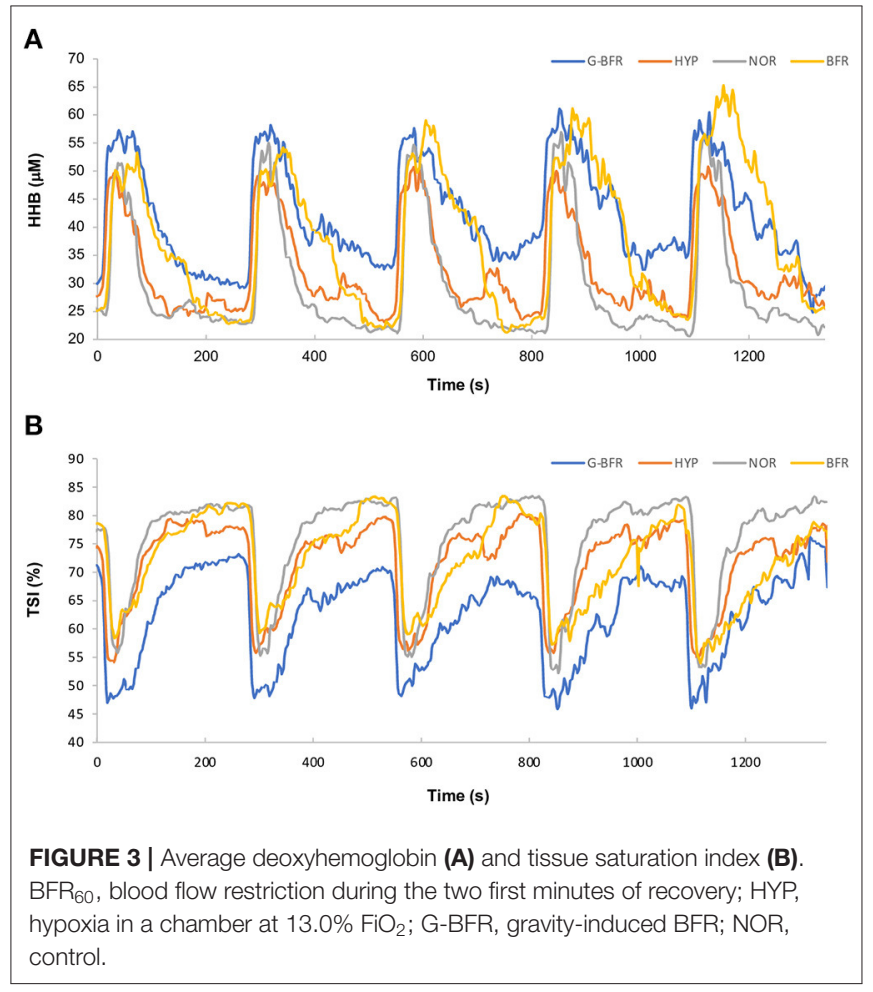

0.5, 0.7, and 1.0; $\left[\mathrm{O}_{2} \mathrm{Hb}\right]_{\min }: d=0.7,1.0$, and $1.2,\left[\mathrm{O}_{2} \mathrm{Hb}\right]: d$ $=0.7,1.0$, and 1.2 for NOR, HYP, and G-BFR, respectively), and in NOR compared to G-BFR $\left(p<0.05,\left[\mathrm{O}_{2} \mathrm{Hb}\right]_{\max }\right.$ : $d=$ $\left.0.7 ;\left[\mathrm{O}_{2} \mathrm{Hb}\right]_{\min }: \mathrm{d}=0.8 ;\left[\mathrm{O}_{2} \mathrm{Hb}\right]: d=1.0\right)$. Moreover, $\left[\mathrm{O}_{2} \mathrm{Hb}\right]$ was lower in HYP compared to NOR $(p<0.05, d=-0.5)$. Finally, an effect of the sprint number was found on $\Delta\left[\mathrm{O}_{2} \mathrm{Hb}\right]$ $(p<0.01)$. Values increased between the first sprint and the last two sprints $(p<0.01): 30.5 \pm 2.9,40.8 \pm 6.0$, and $42.3 \pm$ $3.9 \mu \mathrm{M}$, respectively.

\section{Recovery Periods}

During the first $2 \mathrm{~min}$ of recovery, $\Sigma \mathrm{VT}, \Sigma \mathrm{VO}_{2}$, and $\Sigma \mathrm{VCO}_{2}$ presented a main effect of condition $(p<0.01)$. $\Sigma \mathrm{VT}$ was lower in G-BFR $(p<0.01, d=-0.8,-1.1$, and -0.4 for NOR, HYP, and 
$\mathrm{BFR}_{60}$, respectively) and higher in HYP $(p<0.01, d=0.4,0.7$, and 1.1 for NOR, $\mathrm{BFR}_{60}$, and G-BFR, respectively) compared to the other conditions, and higher in NOR compared to $\mathrm{BFR}_{60}(p<$ $0.01, d=0.4)$. During this first part of recovery, $\Sigma \mathrm{VO}_{2}$ was lower in $\mathrm{BFR}_{60}$ compared to the other conditions $(p<0.01, d=-1.0$, -0.8 , and -0.7 for NOR, HYP, and G-BFR, respectively) and in G-BFR compared to NOR $(p<0.05, d=-0.3)$. $\Sigma \mathrm{VCO}_{2}$ was also lower in $\mathrm{BFR}_{60}$ compared to the other conditions $(p<0.01, d=$ $-0.5,-0.5$, and -0.6 for NOR, HYP, and G-BFR, respectively). Concerning NIRS data, a main effect of condition was found for TSI $(p<0.01)$. It was lower in G-BFR compared to NOR $(p<0.01, d=-1.5)$. Oxyhemoglobin concentration $\left(\left[\mathrm{O}_{2} \mathrm{Hb}\right]\right)$, deoxyhemoglobin concentration ([HHb]), and the difference of hemoglobin concentration $(\Delta[\mathrm{tHb}])$ showed a main effect of condition $(p<0.05)$. $\left[\mathrm{O}_{2} \mathrm{Hb}\right]$ was higher for $\mathrm{BFR}_{60}$ than G-BFR $(p<0.05, d=0.9)$. [HHb] was higher in $\mathrm{BFR}_{60}$ compared to NOR during this period $(p<0.05, d=1.1) . \Delta[\mathrm{tHb}]$ was higher in $\mathrm{BFR}_{60}$ compared to the other conditions $(p<0.05, d=0.9$, 1.6, and 1.5 for NOR, HYP, and G-BFR, respectively).

During the last $2 \mathrm{~min}$ of recovery, $\Sigma \mathrm{VT}, \Sigma \mathrm{VO}_{2}$, and $\Sigma \mathrm{VCO}_{2}$ showed a main effect of condition $(p<0.01)$. $\Sigma \mathrm{VT}$ and $\Sigma \mathrm{VO}_{2}$ were lower in G-BFR compared to the other conditions $(p<0.01$, $\Sigma \mathrm{VT}: d=-0.8,-0.8$, and $-0.9, \Sigma \mathrm{VO}_{2}: d=-0.8 ;-0.9$ and -1.4 for NOR, HYP, and $\mathrm{BFR}_{60}$, respectively). Of note, $\Sigma \mathrm{VO}_{2}$ and $\Sigma \mathrm{VCO}_{2}$ were higher in $\mathrm{BFR}_{60}$ compared to the other conditions $\left(p<0.01, \Sigma \mathrm{VO}_{2}: \mathrm{d}=0.5,0.5\right.$, and $1.4 ; \Sigma \mathrm{VCO}_{2}: d=0.6,0.9$, and 1.0 for NOR, HYP, and G-BFR, respectively). Furthermore, a main effect of condition was observed on TSI, TSI $\min _{\min }$, $[\mathrm{tHb}]_{\max }$, and $[\mathrm{HHb}]_{\max }(p<0.05)$. As previously, TSI was lower in GBFR compared to NOR $(p<0.05, d=-1.2)$. Detailed results are presented in Tables 1, 2, Figures 2, 3.

\section{DISCUSSION}

The aim of the present study was to compare the acute effects of systemic hypoxia, $B_{6 R}$, and G-BFR on mechanical output, cardiorespiratory responses, and $\mathrm{O}_{2}$ muscle extraction during SIT exercises in healthy moderately trained men. The main results were that SIT associated with G-BFR displayed lower mechanical and cardiorespiratory responses than the other modalities. G-BFR also showed lower skeletal muscle oxygenation. $\mathrm{BFR}_{60}$ induced greater blood accumulation within working muscles compared to the other conditions. Moreover, $\mathrm{HYP}$ at $13 \% \mathrm{FiO}_{2}$ and G-BFR increased local hypoxia within the working muscles, with a higher level of hypoxia observed for G-BFR.

The primary results showed that $\mathrm{SpO}_{2}$ was lower for HYP throughout the exercise session and decreased at the end of the session (i.e., sprints four and five) for all the conditions. According to Fick's law of diffusion, systemic hypoxemia occurred in HYP because alveolo-capillary oxygen pressure difference decreases under hypoxic conditions. Consequently, $\dot{V} E_{\text {peak }}$ increased in HYP, as a compensatory response aiming to maintain oxygen consumption. In accordance, previous studies observed that the magnitude of the ventilatory response appears a critical factor of performance during a Wingate test in hypoxia
(Fallon et al., 2015). Indeed, the authors showed that ventilation was elevated at the beginning and throughout the exercise when $\mathrm{F}_{\mathrm{i}} \mathrm{O}_{2}$ was decreased from 20.9 to $10 \%$ (Fallon et al., 2015). However, the augmentation of ventilation observed in the present study was not sufficient, as total and peak oxygen uptake were still lower in HYP compared to NOR. In addition, mean ventilation was higher in HYP during the first period of recovery. Hypoxemia had an impact on muscle oxygenation parameters. Specifically, oxyhemoglobin concentration during sprints was lower in HYP compared to NOR. Therefore, as total work was the same between the conditions, except for G-BFR, this suggests that the anaerobic metabolism is more solicited in $\mathrm{HYP}$. Furthermore, we analyzed $\mathrm{SpO}_{2}$ during recovery when the cuffs were applied and did not observe any difference between the conditions. Similarly, no effect was detected in the study from Willis et al. (2019) using continuous BFR during the RSH protocol at $45 \%$ of arterial occlusive pressure.

In the current protocol, fatigue increased progressively throughout the session as performance variables (i.e., peak and mean power, time to reach mean power) were altered, and HR increased with the repetition of sprints. On the other hand, $\Sigma \mathrm{VO}_{2}$ and $\mathrm{V}_{2 \text { peak }}$ were lower during the first sprint probably because metabolic demands are ensured by the anaerobic metabolism (i.e., phosphocreatine hydrolysis and glycolysis). Interestingly, $\dot{\mathrm{V}}_{2 \text { peak }}$ was higher in NOR compared to the other conditions, which is in accordance with a study from Willis et al. comparing NOR and BFR (Willis et al., 2018). Furthermore, no effect of the condition or the number of sprints was found on FI. A study by Fallon and coworkers (Fallon et al., 2015) observed higher FI in HYP but their study included a single sprint of $30 \mathrm{~s}$ with a higher level of hypoxia $\left(\mathrm{FiO}_{2}=10 \%\right)$. In the current study, the $\mathrm{BFR}_{60}$ application induced a decrease in gas exchange rates, which increased after its release. This phenomenon has already been observed during partial occlusion in dogs where oxygen consumption was decreased during BFR application and increased when pressure was removed. These responses relied on blood flow, which means that BFR causes vascular resistance (Fales et al., 1962). Importantly, in the current study, BFR was applied for the first 2 min of recovery. This probably delayed and reduced overall recovery, which may influence in turn performance, energy system usage, and metabolite accumulation in the following sprints. The BFR protocol used in the present study was applied during recovery periods between exercise bouts and as such has a different effect to the continuous application that may be seen during other studies. For HYP, performance variables were unaffected but average and maximal oxygen consumption and muscular oxyhemoglobin availability decreased. For all conditions, peak and average power decreased after each sprint excepting between sprints four and five where values were similar. Time to reach peak power increased between the three first sprints and sprint five. RPE increased throughout the exercises. Altogether, these data suggest that during SIT, both BFR and HYP enhance cellular stress (i.e., metabolite accumulation and/or hypoxia) without affecting total work during the training sessions.

Importantly, several cardiorespiratory parameters (i.e., $\Sigma \mathrm{VT}$, $\dot{\mathrm{V}} \mathrm{E}_{\text {peak }}, \dot{\mathrm{V}} \mathrm{E}_{\text {min }}, \Delta \dot{\mathrm{VE}}, \Sigma \mathrm{VO}_{2}, \Sigma \mathrm{VCO}_{2}, \mathrm{HR}, \mathrm{HR}_{\text {peak }}, \mathrm{HR}_{\text {min }}$, 
$\Delta \mathrm{HR}$ ), mean TSI (exercise and recoveries), $\mathrm{TSI}_{\max }$ and $\mathrm{TSI}_{\min }$ during exercise and/or recovery were lower for G-BFR compared to the other conditions. Of note, during sprints, the supine position induced lower mean, maximal, and minimal tissue saturation. Concerning training data, RPE appeared lower for G-BFR compared to $\mathrm{BFR}_{60}$ and HYP, peak power was lower compared to NOR, and time to achieve peak power was higher compared to $\mathrm{BFR}_{60}$ and HYP. Average power also appeared lower for G-BFR compared to the other conditions. Overall, these data are consistent with the literature. Indeed, an important decrease in strength of the knee flexor and extensor muscles is inherent to the supine vs. seated position (Houtz et al., 1957). Moreover, maximal exercise performance (such as maximal work rate, $\dot{\mathrm{V}}_{2 \max }, \dot{\mathrm{V}} \mathrm{E}_{\max }$, and $\mathrm{HR}_{\max }$ ) during an incremental test has been shown to be impaired in supine position compared to upright cycle position, probably due to a lower perfusion (Hughson et al., 1991). Our results are consistent with the recent study of Preobrazenski and colleagues who showed a decrease in muscle oxygenation in their G-BFR model compared to the control group. However, they reported a higher RPE with GBFR whereas we obtained the opposite result. This could be explained by the difference in exercise modality (aerobic vs. repeated all-out exercises). Altogether, the G-BFR condition may alter biomechanical and cardiorespiratory responses. However, G-BFR induced lower mean tissue oxygenation. Thus, G-BFR seems to induce specific stress compared to the other modalities.

Concerning $\mathrm{BFR}_{60}$ and $\mathrm{HYP}$, present data indicate no difference with NOR for mechanical outcomes. These results are in line with the data from Willis et al. (2019) who compared NOR, BFR, and HYP during an RST protocol. Indeed, the authors found no difference in mean power, mean ventilation,

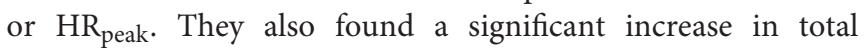
hemoglobin concentration with BFR (constant pressure of $45 \%)$. The present results also suggest that $\mathrm{BFR}_{60}$ promotes higher blood accumulation within working muscles than the other conditions, meaning that $\mathrm{BFR}_{60}$ may additionally affect training adaptations by confining metabolites. Importantly, BFR was suggested to induce greater neuromuscular fatigue while HYP produces central fatigue by impairing corticospinal excitability because of cerebral deoxygenation (Willis et al., 2018; Peyrard et al., 2019). During BFR application, deoxyhemoglobin concentration was higher and oxyhemoglobin content was not different compared to NOR. This result means that a partial occlusion $(60 \%)$ has a greater impact on venous return than on arterial blood supply. Of note, power output strongly decreases for G-BFR, which may explain in turn the decrease of $H R$ values while it should be increased with higher venous return. Furthermore, enhanced deoxyhemoglobin concentration matches the blood accumulation (increased $\Delta[\mathrm{tHb}]$ ) observed during BFR application. Its release induces bigger changes in oxyhemoglobin, deoxyhemoglobin, and total hemoglobin concentrations compared to the other conditions at the same period. On the contrary, our results suggest that G-BFR does not have a significant impact on venous return, as recently demonstrated by another group during aerobic exercises (Preobrazenski et al., 2021). During sprints, TSI was higher for $\mathrm{BFR}_{60}$ compared to the other conditions, due to an increased oxyhemoglobin concentration $\left(\left[\mathrm{O}_{2} \mathrm{Hb}\right]\right)$. On the other hand, peak and mean $\mathrm{HR}$ were lower in $\mathrm{BFR}_{60}$ during the exercises. Interestingly, HYP and G-BFR induced greater local hypoxia within skeletal muscles, which was more prominent in G-BFR when considering both exercise bouts and recovery periods. Thus, G-BFR would represent an alternative to HYP to promote additional hypoxic stress within skeletal muscles cells. Additional studies are needed to compare the chronic effects of these conditions on both cellular adaptations to training and gains in muscle performance, especially because G-BFR lowers power output during sessions.

Furthermore, some limits must be acknowledged. First, the modalities of $\mathrm{HYP}, \mathrm{BFR}_{60}$, and G-BFR conditions were established according to preliminary work that allowed to identify the maximal stress that could be tolerated by the participants in combination with the SIT protocol of this study. Thus, further studies are needed to evaluate more precisely the nature and the degree of the stress generated by these conditions. On the other hand, $\mathrm{BFR}_{60}$ was the only stress which was not continuously applied. In such a situation, the BFR application still had a delayed impact on muscle oxygenation parameters during sprints. However, based on preliminary work, this mode of BFR has been chosen to set maximal stress that could be tolerated in this population in combination with the SIT protocol. Another limit can be that participants were moderately trained individuals, and it was a demanding protocol. This also makes it difficult to generalize the results, especially to untrained populations for which these training methods would be hard to complete. Moreover, TSI measurements cover a limited zone of the vastus lateralis muscle thus interpretations of the results at the whole muscle are to be considered with these limitations. Finally, the present study did not include women athletes. Indeed, some adaptations such as cardiovascular responses differ between men and women (Patel et al., 2021), thus introducing variability. Additional works are needed to compare outcomes according to gender because women remain underrepresented in sport science literature.

\section{CONCLUSIONS}

In conclusion, this study conducted in healthy moderately trained men showed that a session of SIT in combination with GBFR showed lower mechanical, cardiorespiratory responses, and skeletal muscle oxygenation than the other conditions. Another important insight is that both SIT protocols conducted under $\mathrm{HYP}$ at $13 \% \mathrm{FiO}_{2}$ and G-BFR amplified local hypoxia within the working muscles. Importantly, a higher level of hypoxia was found with G-BFR when considering the measurement of the entire exercise session (i.e., exercise bouts and recovery periods). Furthermore, a single session of SIT associated with $\mathrm{BFR}_{60}$ promotes higher blood accumulation within working muscles compared to the other exercise modes. This suggests that $\mathrm{BFR}_{60}$ may additionally or differentially affect cellular homeostasis. Thus, each condition generates specific stress and further studies are needed to better understand subsequent consequences on long-term adaptation. 


\section{PERSPECTIVES}

Further studies are needed (i) to compare the effects of these protocols on-field performance, (ii) to evaluate more precisely the degree of stress generated by each condition, even if high values for both $\mathrm{BFR}_{60}$ and hypoxia have been used based on literature for this kind of exercise, and (iii) to examine mechanistic insight since the mechanisms of action may be different. Investigations of the involvement of different cellular pathways, for example, those involved in mitochondrial adaptations (e.g., the axis of peroxisome proliferator-activated receptor-gamma coactivator 1 -alpha) are needed to improve our knowledge about the molecular benefits of these training methods. These research directions are important because they may help to improve the ability to develop more efficient hypoxic or BFR training modalities and to improve skeletal muscle function and whole-body metabolism. Finally, adding stress to training would promote adaptations in the long term, at least when recovery processes are sufficient.

\section{DATA AVAILABILITY STATEMENT}

The raw data supporting the conclusions of this article will be made available by the authors, without undue reservation.

\section{REFERENCES}

Adreani, C. M., and Kaufman, M. P. (1998). Effect of arterial occlusion on responses of group III and IV afferents to dynamic exercise. J. Appl. Physiol. 84, 1827-1833. doi: 10.1152/jappl.1998.84.6.1827

Baayen, R. H., Davidson, D. J., and Bates, D. M. (2008). Mixed-effects modeling with crossed random effects for subjects and items. J. Mem. Lang. 59, 390-412. doi: 10.1016/j.jml.2007.12.005

Barnett, C., Carey, M., Proietto, J., Cerin, E., Febbraio, M. A., and Jenkins, D. (2004). Muscle metabolism during sprint exercise in man: influence of sprint training. J. Sci. Med. Sport 7, 314-322. doi: 10.1016/S1440-2440(04)80026-4

Bishop, D. J., Botella, J., Genders, A. J., Lee, M. J.-C., Saner, N. J., Kuang, J., et al. (2019). High-intensity exercise and mitochondrial biogenesis: current controversies and future research directions. Physiol. Bethesda Md 34, 56-70. doi: $10.1152 /$ physiol.00038.2018

Boisgontier, M. P., and Cheval, B. (2016). The anova to mixed model transition. Neurosci. Biobehav. Rev. 68, 1004-1005. doi: 10.1016/j.neubiorev.2016.05.034

Brechbuhl, C., Brocherie, F., Willis, S. J., Blokker, T., Montalvan, B., Girard, O., et al. (2020). On the use of the repeated-sprint training in hypoxia in tennis. Front. Physiol. 11:588821. doi: 10.3389/fphys.2020.588821

Brocherie, F., Girard, O., Faiss, R., and Millet, G. P. (2017). Effects of repeatedsprint training in hypoxia on sea-level performance: a meta-analysis. Sports Med. Auckl. NZ 47, 1651-1660. doi: 10.1007/s40279-017-0685-3

Buchheit, M., and Laursen, P. B. (2013a). High-intensity interval training, solutions to the programming puzzle: Part I: cardiopulmonary emphasis. Sports Med. Auckl. NZ 43, 313-338. doi: 10.1007/s40279-013-0029-x

Buchheit, M., and Laursen, P. B. (2013b). High-intensity interval training, solutions to the programming puzzle. Part II: anaerobic energy, neuromuscular load and practical applications. Sports Med. Auckl. NZ 43, 927-954. doi: 10.1007/s40279-013-0066-5

Burgomaster, K. A., Heigenhauser, G. J. F., and Gibala, M. J. (2006). Effect of short-term sprint interval training on human skeletal muscle carbohydrate metabolism during exercise and time-trial performance. J. Appl. Physiol. Bethesda Md 1985 100, 2041-2047. doi: 10.1152/japplphysiol.01220.2005

Burgomaster, K. A., Howarth, K. R., Phillips, S. M., Rakobowchuk, M., Macdonald, M. J., McGee, S. L., et al. (2008). Similar metabolic adaptations during exercise

\section{ETHICS STATEMENT}

The experimental protocol was approved by the Local Ethics Committee (VD-2021-00597). The patients/participants provided their written informed consent to participate in this study.

\section{AUTHOR CONTRIBUTIONS}

RS, FB, and AS: conceived and designed the experiments, analyzed the data, designed the tables and figures, wrote the manuscript, contributed reagents, materials, and analysis tools. RS, HB, and FB: performed the experiments. All authors read the manuscript and approved the final version.

\section{FUNDING}

An internal Bonus Qualité Recherche from the University of Perpignan Via Domitia was obtained to perform this study.

\section{ACKNOWLEDGMENTS}

The authors thank all the participants for their motivation and Pr. Holdel Passo for helpful discussion.

after low volume sprint interval and traditional endurance training in humans. J. Physiol. 586, 151-160. doi: 10.1113/jphysiol.2007.142109

Durnin, J. V. G. A., and Womersley, J. (1974). Body fat assessed from total body density and its estimation from skinfold thickness: measurements on 481 men and women aged from 16 to 72 Years. Br. J. Nutr. 32, 77-97. doi: 10.1079/BJN19740060

Faiss, R., Girard, O., and Millet, G. P. (2013). Advancing hypoxic training in team sports: from intermittent hypoxic training to repeated sprint training in hypoxia. Br. J. Sports Med. 47, i45-i50. doi: 10.1136/bjsports-2013092741

Fales, J. T., Heisey, S. R., and Zierler, K. L. (1962). Blood flow from and oxygen uptake by muscle, during and after partial venous occlusion. Am. J. Physiol. 203, 470-474. doi: 10.1152/ajplegacy.1962.203.3.470

Fallon, S., Belcoe, A., Shawcross, C., May, A., Monteverde, C., and McCann, D. (2015). Elite female athletes' ventilatory compensation to decreased inspired O2 during the wingate test. Res. Q. Exerc. Sport 86, 182-189. doi: 10.1080/02701367.2014.983448

Gibala, M. J., Little, J. P., van Essen, M., Wilkin, G. P., Burgomaster, K. A., Safdar, A., et al. (2006). Short-term sprint interval versus traditional endurance training: similar initial adaptations in human skeletal muscle and exercise performance. J. Physiol. 575, 901-911. doi: 10.1113/jphysiol.2006. 112094

Gueorguieva, R., and Krystal, J. H. (2004). Move over ANOVA: progress in analyzing repeated-measures data and its reflection in papers published in the Archives of General Psychiatry. Arch. Gen. Psychiatry 61, 310-317. doi: 10.1001/archpsyc.61.3.310

Houtz, S. J., Lebow, M. J., and Beyer, F. R. (1957). Effect of posture on strength of the knee flexor and extensor muscles. J. Appl. Physiol. 11, 475-480. doi: 10.1152/jappl.1957.11.3.475

Hughson, R. L., Cochrane, J. E., and Butler, G. C. (1993). Faster O2 uptake kinetics at onset of supine exercise with than without lower body negative pressure. J. Appl. Physiol. Bethesda Md 1985 75, 1962-1967. doi: 10.1152/jappl.1993.75.5.1962

Hughson, R. L., Xing, H. C., Borkhoff, C., and Butler, G. C. (1991). Kinetics of ventilation and gas exchange during supine and upright cycle exercise. Eur. J. Appl. Physiol. 63, 300-307. doi: 10.1007/BF00233866 
James, C., and Girard, O. (2020). In-season repeated-sprint training in hypoxia in international field hockey players. Front. Sports Act. Living 2:66. doi: 10.3389/fspor.2020.00066

Judd, C. M., Westfall, J., and Kenny, D. A. (2017). Experiments with more than one random factor: designs, analytic models, and statistical power. Annu. Rev. Psychol. 68, 601-625. doi: 10.1146/annurev-psych-122414-033702

MacDougall, J. D., Hicks, A. L., MacDonald, J. R., McKelvie, R. S., Green, H. J., and Smith, K. M. (1998). Muscle performance and enzymatic adaptations to sprint interval training. J. Appl. Physiol. Bethesda Md 1985 84, 2138-2142. doi: 10.1152/jappl.1998.84.6.2138

Mendonca, G. V., Vaz, J. R., Teixeira, M. S., Grácio, T., and Pezarat-Correia, P. (2014). Metabolic cost of locomotion during treadmill walking with blood flow restriction. Clin. Physiol. Funct. Imaging 34, 308-316. doi: 10.1111/cpf.12098

Mitchell, E. A., Martin, N. R. W., Turner, M. C., Taylor, C. W., and Ferguson, R. A. (2019). The combined effect of sprint interval training and postexercise blood flow restriction on critical power, capillary growth, and mitochondrial proteins in trained cyclists. J. Appl. Physiol. 126, 51-59. doi: 10.1152/japplphysiol.01082.2017

Moritani, T., Sherman, W. M., Shibata, M., Matsumoto, T., and Shinohara, M. (1992). Oxygen availability and motor unit activity in humans. Eur. J. Appl. Physiol. 64, 552-556. doi: 10.1007/BF00843767

Muth, C., Bales, K. L., Hinde, K., Maninger, N., Mendoza, S. P., and Ferrer, E. (2016). Alternative models for small samples in psychological research: applying linear mixed effects models and generalized estimating equations to repeated measures data. Educ. Psychol. Meas. 76, 64-87. doi: $10.1177 / 0013164415580432$

Okita, K., Takada, S., Morita, N., Takahashi, M., Hirabayashi, K., Yokota, T., et al. (2019). Resistance training with interval blood flow restriction effectively enhances intramuscular metabolic stress with less ischemic duration and discomfort. Appl. Physiol. Nutr. Metab. 44, 759-764. doi: 10.1139/apnm-2018-0321

Ozaki, H., Brechue, W. F., Sakamaki, M., Yasuda, T., Nishikawa, M., Aoki, N., et al. (2010). Metabolic and cardiovascular responses to upright cycle exercise with leg blood flow reduction. J. Sports Sci. Med. 9, 224-230.

Patel, R., Kemp, C. L., Hafejee, M., Peckham, N., Jain, V., McCann, G. P., et al. (2021). The underrepresentation of females in studies assessing the impact of high-dose exercise on cardiovascular outcomes: a scoping review. Sports Med. Open 7:30. doi: 10.1186/s40798-021-00320-y

Peyrard, A., Willis, S. J., Place, N., Millet, G. P., Borrani, F., and Rupp, T. (2019). Neuromuscular evaluation of arm-cycling repeated sprints under hypoxia and/or blood flow restriction. Eur. J. Appl. Physiol. 119, 1533-1545. doi: 10.1007/s00421-019-04143-4

Preobrazenski, N., Islam, H., Drouin, P. J., Bonafiglia, J. T., Tschakovsky, M. E., and Gurd, B. J. (2020). A novel gravity-induced blood flow restriction model augments ACC phosphorylation and PGC-1 $\alpha$ mRNA in human skeletal muscle following aerobic exercise: a randomized crossover study. Appl. Physiol. Nutr. Metab. Physiol. Appl. Nutr. Metab. 45, 641-649. doi: 10.1139/apnm-20 19-0641

Preobrazenski, N., Islam, H., and Gurd, B. J. (2021). Molecular regulation of skeletal muscle mitochondrial biogenesis following blood flow-restricted aerobic exercise: a call to action. Eur. J. Appl. Physiol. 121, 1835-1847. doi: 10.1007/s00421-021-04709-1

Sanchez, A. M., Candau, R., and Bernardi, H. (2019). Recent data on cellular component turnover: focus on adaptations to physical exercise. Cells 8:542. doi: $10.3390 /$ cells 8060542

Silva, J. C. G., Domingos-Gomes, J. R., Freitas, E. D. S., Neto, G. R., Aniceto, R. R., Bemben, M. G., et al. (2019). Physiological and perceptual responses to aerobic exercise with and without blood flow restriction. J Strength Cond Res. 35, 2479-2485. doi: 10.1519/JSC.0000000000003178

Solsona, R., Pavlin, L., Bernardi, H., and Sanchez, A. M. (2021). Molecular regulation of skeletal muscle growth and organelle biosynthesis: practical recommendations for exercise training. Int. J. Mol. Sci. 22:2741. doi: 10.3390/ijms22052741

Sugaya, M., Yasuda, T., Suga, T., Okita, K., and Abe, T. (2011). Change in intramuscular inorganic phosphate during multiple sets of blood flowrestricted low-intensity exercise. Clin. Physiol. Funct. Imaging 31, 411-413. doi: 10.1111/j.1475-097X.2011.01033.x

Taylor, C. W., Ingham, S. A., and Ferguson, R. A. (2016). Acute and chronic effect of sprint interval training combined with postexercise blood-flow restriction in trained individuals. Exp. Physiol. 101, 143-154. doi: 10.1113/EP085293

Teixeira, E. L., Barroso, R., Silva-Batista, C., Laurentino, G. C., Loenneke, J. P., Roschel, H., et al. (2018). Blood flow restriction increases metabolic stress but decreases muscle activation during high-load resistance exercise. Muscle Nerve 57, 107-111. doi: 10.1002/mus.25616

Willis, S. J., Alvarez, L., Borrani, F., and Millet, G. P. (2018). Oxygenation time course and neuromuscular fatigue during repeated cycling sprints with bilateral blood flow restriction. Physiol. Rep. 6:e13872. doi: 10.14814/phy2.13872

Willis, S. J., Peyrard, A., Rupp, T., Borrani, F., and Millet, G. P. (2019). Vascular and oxygenation responses of local ischemia and systemic hypoxia during arm cycling repeated sprints. J. Sci. Med. Sport 22, 1151-1156. doi: 10.1016/j.jsams.2019.05.001

Conflict of Interest: The authors declare that the research was conducted in the absence of any commercial or financial relationships that could be construed as a potential conflict of interest.

Publisher's Note: All claims expressed in this article are solely those of the authors and do not necessarily represent those of their affiliated organizations, or those of the publisher, the editors and the reviewers. Any product that may be evaluated in this article, or claim that may be made by its manufacturer, is not guaranteed or endorsed by the publisher.

Copyright (c) 2021 Solsona, Berthelot, Borrani and Sanchez. This is an open-access article distributed under the terms of the Creative Commons Attribution License (CC $B Y)$. The use, distribution or reproduction in other forums is permitted, provided the original author(s) and the copyright owner(s) are credited and that the original publication in this journal is cited, in accordance with accepted academic practice. No use, distribution or reproduction is permitted which does not comply with these terms. 